\title{
Soul searching
}

\section{What have advances in neuroscience told us about the mind?}

\author{
Soul Made Flesh: The Discovery \\ of the Brain and How it Changed \\ the World \\ by Carl Zimmer \\ Free Press: 2004.384 pp. $\$ 26$ \\ To be published in the UK by Heinemann \\ in April, $£ 17.99$
}

\section{Rina Knoeff}

The provocative title of this book alone, Soul Made Flesh, made me want to read it. After all, we live in a time where scientists are doing their best to explain all mental phenomena in terms of matter. Advanced medical technology, such as the magnetic resonance imaging (MRI) scan, makes the processes of the brain visible and gives us the feeling that we are gaining an insight into the nature of the mind and its functions. Even more, we are relieved when psychological disorders can be explained in terms of a malfunctioning brain. It enables us to ascribe our depressions and mania to something alien that is not essentially part of our true selves.

Carl Zimmer's fascinating book shows that what we think of as recent developments began in the seventeenth century, when the English anatomist Thomas Willis began dissecting brains in Beam Hall at Oxford. At the beginning of the seventeenth century, the brain was seen as a 'bowl of curds', functioning as a kind of refrigerator to cool the heat of the blood. By the end of the century, thanks to Willis, the brain was studied as the seat of emotions, perception and memory.

Surprisingly enough, Willis is largely unknown. Some might remember him as the discoverer of the so-called 'circle of Willis', a ring of blood vessels at the base of the brain, but it is not widely known that his descriptions of the brain and nerves are at the root of modern neurology. According to Zimmer, this is because John Locke's ideas eclipsed those of Willis. Locke argued that we cannot know much about the inner working of the mind, so we should restrict ourselves to the ideas themselves and how they are confirmed by everyday experience. Willis, on the other hand, showed that the anatomy and chemistry of the brain could reveal the working of the mind - an idea that was dangerous at the time because it smacked of atheism.

Yet, Willis managed to keep out of theological trouble by strictly separating the immortal, rational soul from its bodily counterpart, the sensitive soul. The latter, Willis argued, consists of tiny particles that function as messengers, conveying sense and motion from the brain via the nerves to the rest of the body, and vice versa. Illness

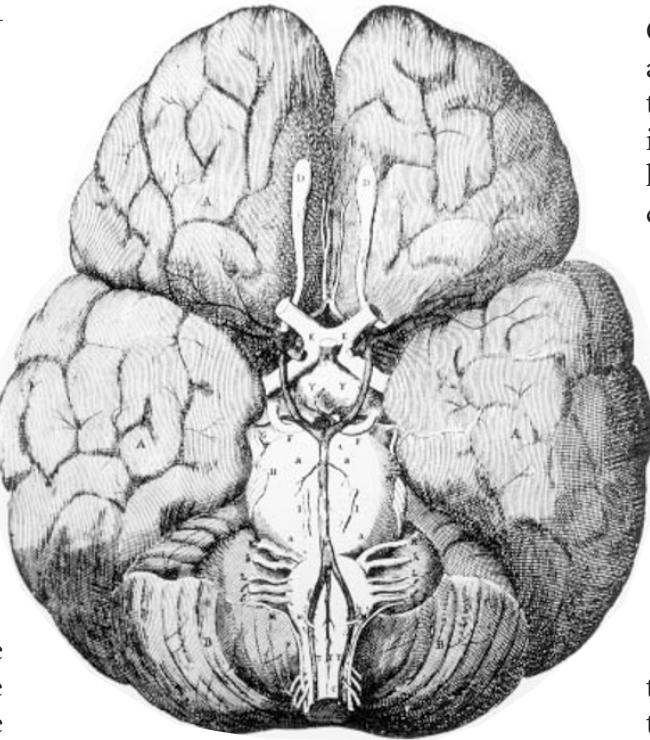

Oxford to a present-day MRI investigation at Princeton University. He introduces us to the philosopher Joshua Green, who is interrogating someone in the machine while he looks at depictions of the brain on his computer screen. The aim of his research is to establish a better understanding of the nature of moral judgements. The soul made flesh - it is an eerie reality of modern neurological and philosophical research. To my taste, Zimmer does not sufficiently distinguish Willis's concept of soul from its twentieth-century counterpart. For Willis, the concept of soul referred to the processes of life at large, whereas nowadays we associate the soul with mental functions.

In the seventeenth century, people tried to cure diseases of the mind by manipulating the brain and nervous system. It is this continuity of concern that makes Zimmer's book such a fascinating read.

Christopher Wren's drawing of the brain shows blood vessels discovered by Thomas Willis.

is caused by miscommunication and damage to the brain. Most notably, Willis was able to explain psychological illnesses and disabilities resulting from brain damage.

Zimmer's book is fascinating, not least because it provides a vivid picture of the world of which Willis was a part. Not only does he describe the smells of seventeenthcentury Oxford in a way that transports you right there, but he also convincingly explains Willis's work in its political and religious contexts. We meet Oliver Cromwell and his puritan followers as well as King Charles II and his curiosity for the new experimental natural philosophy. Thomas Hobbes and Lady Anne Conway also figure in the story. Last, but not least, the reader is made part of the audience watching the often outrageous and crazy experiments of the so-called Oxford experimental circle - a group of natural philosophers busy with topics as diverse as submarines, blood transfusions, spacecraft and vacuum pumps.

Historians of science have written extensively about seventeenth-century English natural philosophy. Yet making sense of the diverse experiments of the Royal Society and its forerunners remains a tricky enterprise. Historians usually focus on one or two philosophers, a series of experiments or a particular philosophical movement. Zimmer's book is remarkable in that it offers a multifaceted picture of the world and work of Willis. In so doing he has managed to make sense of Willis's ideas in turbulent times of revolution, plague and fire.

In the final chapter, Zimmer moves from the dissection room in seventeenth-century
Rina Knoeff is in the Faculty of Arts and Culture, Maastricht University, 6200 MD Maastricht, the Netherlands.

\section{Systematic survey of the spheres}

\section{Physics of the Solar System: Dynamics and Evolution, Space Physics, and Spacetime Structure by Bruno Bertotti, Paolo Farrinella \& David Vokrouhlický}

Kluwer: 2003.701 pp. \$229, £128, €209

\section{Doug Hamilton}

Progress in our understanding of the Solar System has been extremely rapid over the past decade. It has been fuelled by the discoveries of extrasolar planets, the Kuiper belt and myriad distant planetary satellites, by spacecraft fly-bys of asteroids and comets, by the in situ exploration of Mars, and by the advent of fast computers and efficient numerical algorithms. To the great benefit of students and practitioners alike, the appearance of advanced textbooks has been almost as rapid. The most recent offering is Physics of the Solar System, a wellwritten and comprehensive overview of the diverse bodies that surround the Sun and of the intricate interplay between them.

Physics of the Solar System is much broader in scope than two other recent advanced textbooks, Solar System Dynamics by Carl Murray and Stanley Dermott (Cambridge University Press, 2000) and Alessandro 
Morbidelli's Modern Celestial Mechanics (Taylor \& Francis, 2002). These titles focused primarily on orbital dynamics, a topic that accounts for only about half of Physics of the Solar System, which manages to cover it clearly and succinctly by outlining many derivations rather than providing full details.

Preceding the orbital dynamics is an opening section that emphasizes the physical states of planetary bodies, including their rotation, gravitational fields, tidal distortions and heat budgets, and another that focuses on magnetospheres and atmospheres. The volume concludes with several chapters devoted to issues relevant for artificial satellites. The book is more focused and deeper than Planetary Sciences (Cambridge University Press, 2001) by Imke de Pater and Jack Lissauer, but is less broad, containing no chemistry and only a limited discussion of geological topics.

Physics of the Solar System is tightly written, fun to read and should appeal to experts in the field and new graduate students alike. Within its covers abound a wealth of interesting and little-known nuggets of planetary lore that, although available in the scientific literature, have not appeared in an accessible text before. For instance, it is well known that planets move along elliptical paths around the Sun and that these orbits precess (or rotate) slowly in space under the influence of weak gravitational perturbations from the other planets. What is less widely known is that these elliptical orbits do not always precess independently; those of Jupiter and Uranus, for example, oscillate about one another, while precessing at a common rate.

The book has a nice introduction to planetary atmospheres that starts from the concept of hydrostatic equilibrium. The authors show that an atmosphere of constant temperature continually loses gas molecules to space, and they make an analogy to the solar wind. Magnetic fields are treated in greater depth than is usual for a planetary textbook, but without the overwhelming detail of a text on plasma physics. The authors keep their focus on the Solar System by pointing out, for instance, that the energy source that powers Earth's magnetic dynamo is thought to arise from heat released as Earth's core solidifies. The lack of ongoing core solidification in Venus, rather than the slow rotation of our sister planet, is the preferred explanation for its lack of an active dynamo.

The authors provide a clear and insightful discussion of planetary gravitational fields. They describe how the dipole terms of an expansion of a gravitational field vanish with the choice of the centre of mass as the origin, and show that two of the five quadrupole terms disappear when the $z$-axis is chosen to point along the spin axis. They show that although geostationary satellites drift slightly relative to a fixed point on Earth's equator,

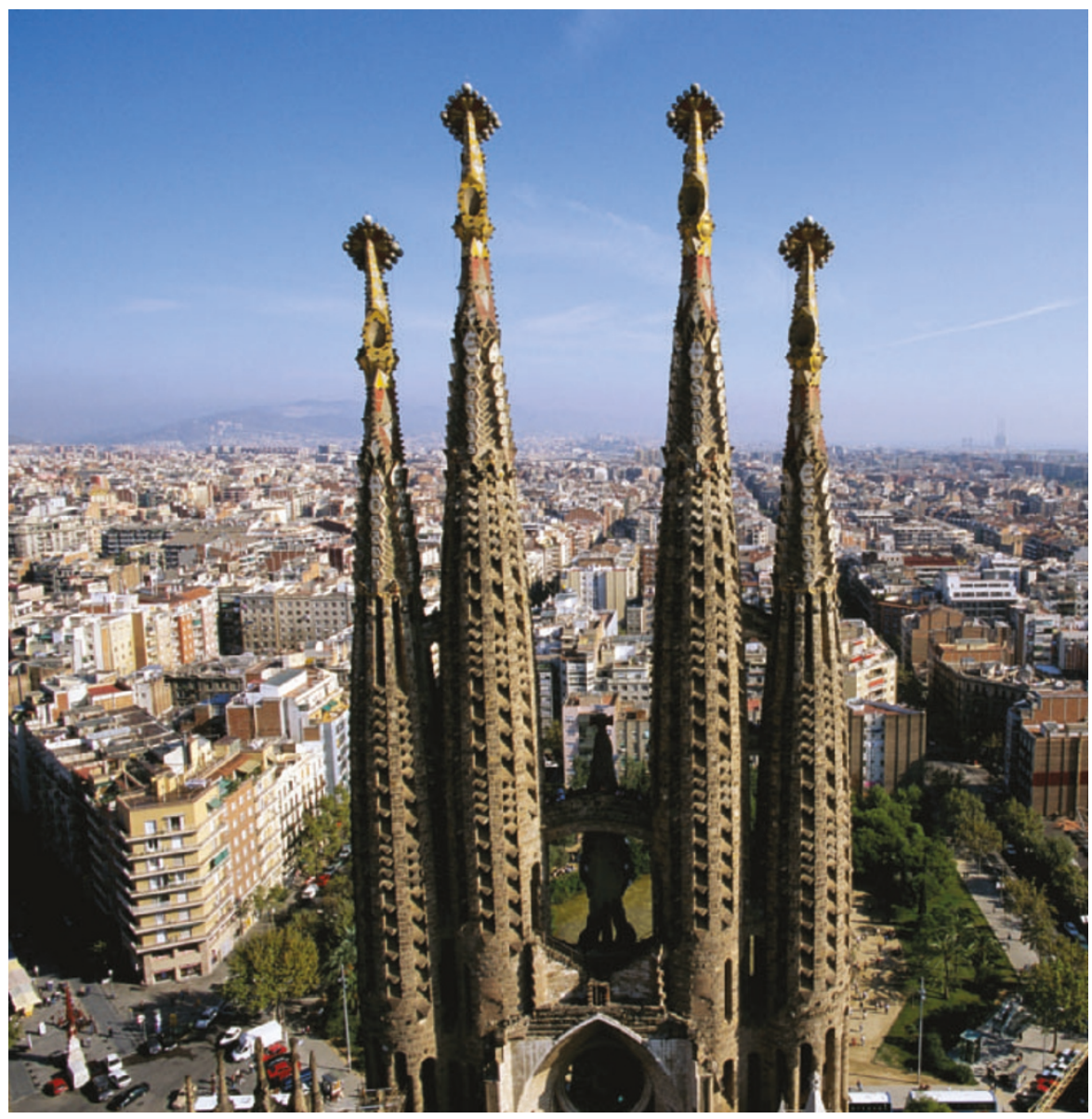

Inspiring: Antoni Gaudí's unfinished church, the Sagrada Familia, dominates the Barcelona skyline.

there are two stable longitudes where this drift vanishes, thanks to a 1:1 resonance with the geopotential. Theselongitudes are defined by the minimum equatorial diameter of Earth, which pierces our planet near the Maldives and the Galapagos - true islands of stability in Earth's chaotic seas.

There is also a beautiful presentation of orbital-perturbation theory. It begins with a derivation of the perturbation equations of celestial mechanics based on changes to energy and angular momentum. Subsequent discussion applies the equations to the examples of atmospheric drag and Earth's quadrupole field. I follow the same scheme when teaching my undergraduate orbitaldynamics class.

Each chapter comes with a generous set of useful and challenging problems, making the text appropriate for a graduate-level course on the Solar System. One minor quibble is that the chapter on the space-time structure of the Solar System, which is a brief introduction to the general theory of relativity, is less well linked to the book's other topics than one expects from the book's subtitle. Even so, I strongly recommend making space on your shelf and time in your schedule for this lively, interesting and authoritative volume.

Doug Hamilton is in the Department of

Astronomy, University of Maryland,

College Park, Maryland 20742-2421, USA.

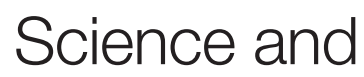
the city

Walks around the Scientific World of Barcelona

by Xavier Duran \& Merce Piqueras

Ajuntament de Barcelona: 2003. 362 pp.

$€ 19$. www.bcn.es/publicacions

\section{Jacqueline Reynolds}

and Charles Tanford

The Spanish city of Barcelona dates back to the third century BC. It expanded greatly under Roman occupation from $27 \mathrm{BC}$ to $\mathrm{AD} 14$ but remained largely unaffected by the Moorish invasion that left its mark on so much of the southern Iberian peninsula. Barcelona's strategic location on the Mediterranean trade routes helped it to develop into an important industrial and commercial centre. Traditional guide books emphasize the city's beautiful architecture, much of it designed by Antoni Gaudí, along with the wealth of museums, the artistic tradition, the famous football team and the hosting of the 1992 Olympic Games. Knowledgeable travellers will probably associate the painters Pablo Picasso would make any connection with science, and Salvador Dalí with the city, but few 